\title{
Advances in magnetic resonance imaging of endometrial cancer
}

\author{
Evis Sala • Andrea Rockall • Rahel A. Kubik-Huch
}

Received: 30 September 2010 / Accepted: 30 October 2010/Published online: 27 November 2010

(C) European Society of Radiology 2010

Endometrial cancer is the most commonly diagnosed gynaecological malignancy in affluent societies [1]. It occurs most frequently in white women, with peak incidence between ages 55 and 65. Risk factors include unopposed oestrogen intake, use of tamoxifen, nulliparity, obesity, and diabetes. The incidence of endometrial cancer in the United Kingdom has increased by more than $40 \%$ between 1993 and 2007. This significant rise is predominantly due to a large increase in incidence in women aged 60-79 [1]. These trends are very similar for most European countries [2]. The increase in prevalence of obesity and decreases in fertility may partly account for the observed rapid increase in incidence and imply that endometrial cancer in postmenopausal women will become a more substantial public health problem in the future.

Prognosis depends on a number of factors, including stage, depth of myometrial invasion, lymphovascular invasion, nodal status and histological grade. Depth of myometrial invasion is the single most important morpho-

\section{E. Sala}

Addenbrooke's Hospital, University Department of Radiology,

Box 218, Level 5, Hills Road,

Cambridge CB2 0QQ, UK

e-mail: es220@radiol.cam.ac.uk

\section{A. Rockall}

Cancer Imaging, Department of Radiology,

St Bartholomew's Hospital,

West Smithfield,

London EC1A 7ED, UK

e-mail: Andrea.Rockall@bartsandthelondon.nhs.uk

\section{R. A. Kubik-Huch ( $\square)$}

Institute of Radiology, Head of the Department of Medical

Services, Kantonsspital Baden,

Im Ergel CH-5404, Baden, Switzerland

e-mail: rahel.kubik@ksb.ch logic prognostic factor, correlating with tumour grade, lymph node metastases and overall patient survival. Incidence of lymph node metastases increases from 3\% with superficial myometrial invasion (stage IA) to $46 \%$ with deep myometrial invasion (stage IB) [3]. Preoperative knowledge of these factors is crucial in tailoring the surgical approach. The histological grade can be determined at endometrial sampling, whereas depth of myometrial invasion can only be assessed preoperatively by MRI. Therefore, MRI can assist in preoperative assessment and treatment planning by accurately predicting depth of myometrial invasion, cervical stromal invasion and lymph node involvement. This information allows selection of patients for pelvic or para-aortic lymph node sampling whilst obviating the need for surgery in patients with low risk disease. MRI can also provide additional useful information such as uterine size, tumour volume, ascites and adnexal pathology which in turn may determine whether the surgical approach is transabdominal, transvaginal or laparoscopic.

Lymphadenectomy in early (stage I) endometrial cancer remains a controversial issue. Two large prospective multicentre studies investigated whether pelvic lymphadenectomy could improve survival of women with endometrial cancer $[4,5]$. Both studies reported no benefit in overall or recurrence free survival in the patients randomized to lymphadenectomy. Conversely, the SEPAL study [6] showed that in patients with intermediate or high risk of endometrial cancer recurrence, combined pelvic and paraaortic lymphadenectomy reduced the risk of death compared with pelvic lymphadenectomy alone. The authors acknowledged that MRI is an important factor for predicting lymph node metastasis, and in combination with tumour grade and histology could be helpful to discriminate patients with very low risk of recurrence. Therefore, 
preoperative accurate assessment of depth of myometrial invasion by MRI remains an important factor in surgical planning [7].

Functional imaging by means of dynamic multiphase contrast-enhanced magnetic resonance imaging (DCEMRI) and diffusion weighted magnetic resonance imaging (DW-MRI) are becoming part of the standard imaging protocols for staging patients with endometrial cancer. This is mainly due to the limitations of morphologic imaging, particularly in the accurate assessment of the depth of myometrial invasion especially in the presence of pitfalls such as loss of junctional zone definition (very common in post-menopausal patients), poor tumour to myometrium contrast, myometrial compression by polypoid tumour, leiomyomas, and adenomyosis. To date, it has been accepted that the combination of DCE-MRI and T2W images offers a 'one-stop' examination with the highest efficacy for staging of patients with endometrial cancer $[8$, 9] and this protocol has been implemented by most European cancer centres as well as recommended in the ESUR Endometrial Cancer Staging Guidelines [10]. Nevertheless, there is no consensus in the literature regarding the added value of DCE-MRI in staging of endometrial cancer. Although the majority of the published studies demonstrate that the addition of multiphase DCE-MRI to $\mathrm{T} 2 \mathrm{~W}$ imaging leads to a significant improvement in the accuracy of assessment of the deep myometrial invasion $[8$, 9, 11-15], some authors have found no significant difference $[16,17]$.

In a recent prospective study published in European Radiology in 2010, Rechichi et al. found that DW-MRI was very accurate in assessing myometrial invasion with a sensitivity, specificity, positive and negative predictive value of $84.6 \%, 70.6 \%, 52.4 \%$ and $92.3 \%$. The corresponding values for dynamic gadolinium-enhanced imaging and $\mathrm{T} 2 \mathrm{~W}$ imaging were $69.2 \%, 61.8 \%, 40.9 \%$, $84.0 \%$ and $92.3 \%, 76.5 \%, 60.0 \%, 96.3 \%$ respectively. The interobserver agreement in assessing the depth of myometrial invasion was substantial for both $\mathrm{T} 2 \mathrm{~W}$ and $\mathrm{DW}$ imaging ( $k=0.91$ and 0.74 respectively) and moderate for dynamic imaging $(k=0.45)$. They suggested that DWI could potentially replace dynamic imaging as an adjunct to routine $\mathrm{T} 2 \mathrm{WI}$ for preoperative evaluation of endometrial cancer [18].

The authors are to be commended for the prospective design and detailed histopathological analysis in this study. In addition to the evaluation of the depth of myometrial invasion, the authors estimated the myometrial thickness in all cases. Incorrect evaluation of depth of myometrial invasion was mainly due to over-calling deep invasion. This was found to be the result of a combination of overestimation of tumour invasion into the myometrium and underestimation of myometrial thickness in all three
Fig. 1 Stage IB endometrial cancer. a Sagittal T2W image, (b) axial T2W image, (c) DW image $(b=800)$, (d) ADC map, (e) fusion image of sagittal T2W and DWI, and dynamic contrast-enhanced (f) early and (g) equilibrium phase $\mathrm{T} 1 \mathrm{~W}$ demonstrate a distended endometrial cavity by presence of a large tumour ( $\mathrm{T}$ in $\mathbf{a}-\mathbf{f})$. A Nabothian cyst $(\mathrm{N})$ is seen in the cervix uteri. The tumour shows restricted diffusion in the ADC map (e), whereas the high signal intensity of the Nabothian cyst on the b800 DW image (c) can be attributed to a T2-shine-through effect. While the arterial phase (f) does not provide any additional information, the image acquired in the equilibrium phase $(2 \mathrm{~min}$.) allows a good delineation of the tumour which enhances less than the adjacent myometrium (f). While histologically confirmed myometrial invasion of the ventral uterine wall was correctly diagnosed on T2W (arrows), tumor extent would have been underestimated by DWI alone

pulse sequences. It is perhaps interesting to note that this group previously reported a sensitivity of $87.5 \%$ for contrast enhanced MRI compared with $71.4 \%$ for T2WI. The authors do not comment on this change in their local results. It may be that technical advances have resulted in improvements in $\mathrm{T} 2 \mathrm{~W}$ sequences.

There are a few methodological limitations that the reader must take in account when evaluating the results of this article. Firstly, the DW and dynamic images were acquired in a single plane (axial) whereas the $\mathrm{T} 2 \mathrm{~W}$ images were acquired in three orthogonal planes (sagittal, axial oblique and coronal oblique). This may explain the higher accuracy of T2W images as optimal assessment of depth of myometrial invasion requires evaluation of at least two orthogonal planes.

Secondly, the only dynamic images used for evaluation of the depth of myometrial invasion were those obtained in the portal venous phase whereas the maximum tumour to myometrium contrast is achieved in the equilibrium phase ( 2 min post injection) which is the most optimal phase of enhancement for assessment of the depth of myometrial invasion. This may have resulted in the lower accuracy of dynamic imaging compare to other sequences. One could even argue that since the distinction between tumours confined to the endometrium and those who invade the inner myometrium is no longer of clinical importance, as both categories are classified as stage IA in the revised FIGO staging system [19], the early phases of enhancement (arterial and portal phase) may no longer be needed for staging of endometrial cancer (Fig. 1). Therefore, "static", spin-echo contrast-enhanced imaging may be a suitable alternative to "dynamic" imaging [10].

Thirdly, single $b$-value DW images $(b=500)$ were reviewed in isolation, with no comparison with respective ADC maps. The DW images should always be evaluated together with $\mathrm{ADC}$ maps and other anatomical sequences (e.g. $\mathrm{T} 1 \mathrm{~W}$ and $\mathrm{T} 2 \mathrm{~W}$ images) in order to avoid potential pitfalls in image interpretation such as T2 shine-through, water restriction in normal and non-malignant tissues and lesions with low cellularity (Figs. 1 and 2). Erroneous 

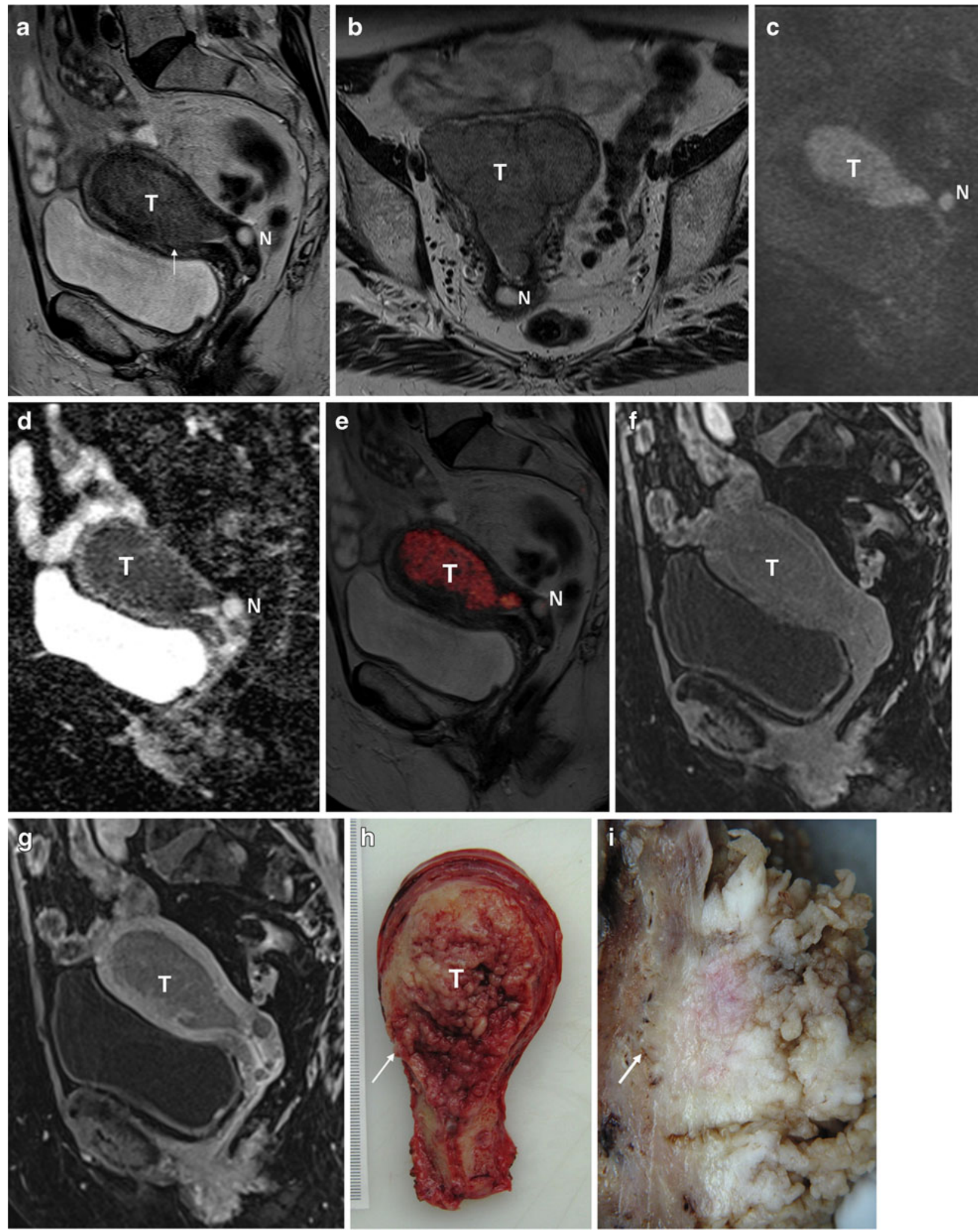

image interpretation may occur if high $b$-value images are viewed in isolation without cross-referencing them to the corresponding ADC maps. High signal intensity on DW images and low signal intensity on the corresponding ADC maps due to restricted diffusion may also occur in areas of retained mucus in an obstructed endometrial cavity, coagulative necrosis and abscesses. In such cases, the anatomic images are very helpful. Some malignant tumours 
Fig. 2 Stage IB endometrial cancer. a Sagittal T2W image, (b) axial $\mathrm{T} 2 \mathrm{~W}$ image, (c) DW image $(b=400)$, (d) DW image $(b=1000)$, and (e) ADC map demonstrate a distended endometrial cavity by presence of tumour ( $\mathrm{T}$ in $\mathbf{a}-\mathbf{e})$ and fluid $(\mathrm{F}$ in a-e). The tumour shows restricted diffusion in the ADC map (e). The low $b$-value DW image (c) and the ADC map (e) allow better differentiation between tumour and fluid within the endometrial cavity. The deep myometrial invasion is perhaps easier to appreciate on DW images (arrows in $\mathbf{c}, \mathbf{d}$ ) compared to $\mathrm{T} 2 \mathrm{~W}$ images (arrows in $\mathbf{a}, \mathbf{b})$
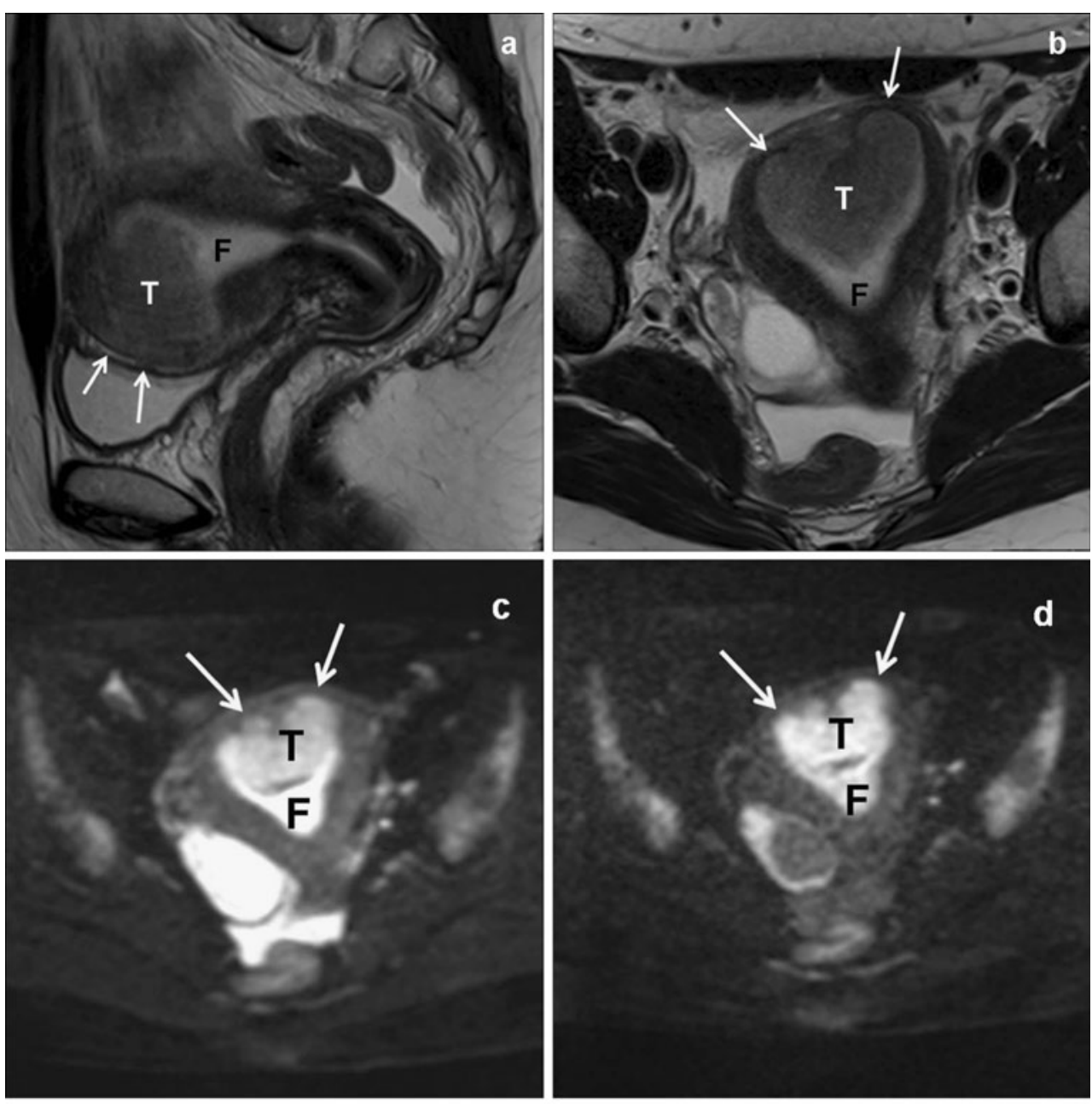

such as well differentiated adenocarcinomas may have little restricted diffusion due to their low cellularity. These may not be visible on DW images and appear bright on ADC maps due to the high ADC value. Correlation with anatomical sequences and potential use of post-processing software for image fusion (T2W and DW images) are therefore very useful in these cases (Fig. 1).
Finally and most importantly, the most useful evaluation would have been comparison of T2W imaging versus T2W imaging + dynamic imaging versus $\mathrm{T} 2 \mathrm{~W}$ imaging $+\mathrm{DW}$ MRI to determine the added value of dynamic imaging and DW-MRI as none of these techniques can be used in isolation. The $\mathrm{T} 2 \mathrm{~W}$ images remain crucial for anatomical reference due to their high spatial resolution which both 
dynamic and DW-MRI sequences lack. This is analogous to co-location of FDG-PET images with CT.

Other studies have also evaluated the accuracy of DWMRI in assessment of myometrial invasion [20-22] and have shown similar results. Shen at al. [20] compared the use of DW-MRI and DCE-MRI in the evaluation of depth of myometrial invasion and found that the diagnostic accuracy was $62 \%$ for DW-MRI compared to $71 \%$ for DCE-MRI. However, both DW-MRI and DCE-MRI images were acquired in the sagittal plane only. This may have contributed to a relatively low accuracy in the evaluation of myometrial invasion as the presence of two orthogonal planes is mandatory for an accurate assessment. DW-MRI was also useful in detection of drop metastases in the cervix or metastatic foci outside the uterus, such as adnexa or peritoneum [20]. Lin et al. [22] demonstrated an accuracy of $88 \%$ in determining the depth of myometrial invasion using fused $\mathrm{T} 2 \mathrm{~W}$ and DW images at $3.0 \mathrm{~T}$.

Detection of nodal metastases remains elusive. Improvements in sensitivity have been reported at $3 \mathrm{~T}$ by Lin et al. [22]. In patients with cervical or uterine cancer, they found that a combination of nodal size and the relative tumour to node ADC resulted in an increase in sensitivity compared with size criteria from 25 to $83 \%$, whilst maintaining very high specificities. However, Nakai et al. reported no difference in diagnostic performance between size criteria and DWI in differentiating benign from malignant nodes in 18 patients with gynaecologic cancer [23]. A more recent study of 259 patients with various pelvic tumours also reported no improvement in sensitivity [24]. Rechichi et al. did not report their findings in relation to nodal appearances.

DW-MRI may also help to differentiate endometrial cancer from normal endometrium. This has limited clinical utility as the diagnosis of endometrial cancer is made at endometrial biopsy, however, pipelle sampling has an error rate and up-staging of the tumour grade may occur in up to $15 \%$ of patients. In these cases, DW-MRI may influence surgical planning by improving pre-operative detection of high grade tumours. The ADC value of endometrial cancer is significantly lower than of endometrial polyps and of normal endometrium $[25,26]$. ADC values can provide useful information in differentiating malignant from benign endometrial lesions with a sensitivity, specificity and accuracy of $85 \%, 100 \%$ and $92 \%$ respectively [25]. High grade endometrial carcinomas have high cellular density and are expected to have lower ADC values (Fig. 1) compare to low-grade ones. Tamai et al. [26] found a trend towards lower ADC values in high grade endometrial cancers. However, one should be aware that there is no reliable cut-off ADC value that is diagnostic of presence of malignancy. Also, tumour necrosis associated with poorly differentiated tumours can have increased ADC values.
In conclusion, DW-MRI is an important imaging technique which may enable accurate staging of endometrial cancer compared to conventional $\mathrm{T} 2 \mathrm{~W}$ images alone (Fig. 1) and obviate the need for dynamic contrast enhanced images. This may be particularly helpful in cases of tumours that are either iso- or hyper-intense relative to the myometrium or when the use of intravenous contrast medium is contra-indicated. This would also result in shorter examination time which leads to a reduction in the cost of MRI. However, the reader must be aware that welldesigned studies are required to directly compare the accuracy of combined T2W \& DW-MRI with combined T2W \& DCE-MRI before one decides to replace dynamic imaging with DW-MRI. DW-MRI cannot replace T2W sequences as it lacks anatomical details that are crucial for accurate assessment of depth of myometrial invasion. Furthermore, the DWI should always be evaluated together with ADC maps and other anatomical sequences (e.g. T1W and $\mathrm{T} 2 \mathrm{~W}$ images) in order to avoid potential pitfalls in image interpretation.

\section{References}

1. http://info.cancerresearchuk.org/cancerstats/types/uterus/incidence/ (date of access: 1. November 2010)

2. Bray F, Dos Santos SI, Moller H, Weiderpass E (2005) Endometrial cancer incidence trends in Europe: underlying determinants and prospects for prevention. Cancer Epidemiol Biomarkers Prev 14:1132-1142

3. Larson DM, Connor GP, Broste SK, Krawisz BR, Johnson KK (1996) Prognostic significance of gross myometrial invasion with endometrial cancer. Obstet Gynecol 88:394-398

4. Benedetti Panici P, Basile S, Maneschi F et al (2008) Systematic pelvic lymphadenectomy vs. no lymphadenectomy in early-stage endometrial carcinoma: randomized clinical trial. J Natl Cancer Inst 100:1707-1716

5. Kitchener H, Swart AM, Qian Q, Amos C, Parmar MK (2009) Efficacy of systematic pelvic lymphadenectomy in endometrial cancer (MRC ASTEC trial): a randomised study. Lancet 373:125136

6. Todo Y, Kato H, Kaneuchi M, Watari H, Takeda M, Sakuragi N (2010) Survival effect of para-aortic lymphadenectomy in endometrial cancer (SEPAL study): a retrospective cohort analysis. Lancet 375:1165-1172

7. Frei KA, Kinkel K, Bonel HM, Lu Y, Zaloudek C, Hricak H (2000) Prediction of deep myometrial invasion in patients with endometrial cancer: clinical utility of contrast-enhanced MR imaging-a meta-analysis and Bayesian analysis. Radiology 216:444-449

8. Manfredi R, Mirk P, Maresca G et al (2004) Local-regional staging of endometrial carcinoma: role of MR imaging in surgical planning. Radiology 231:372-378

9. Sala E, Crawford R, Senior E et al (2009) Added value of dynamic contrast-enhanced magnetic resonance imaging in predicting advanced stage disease in patients with endometrial carcinoma. Int J Gynecol Cancer 19:141-146

10. Kinkel K, Forstner R, Danza FM et al (2009) Staging of endometrial cancer with MRI: guidelines of the European Society of Urogenital Imaging. Eur Radiol 19:1565-1574 
11. Ito $K$, Matsumoto $T$, Nakada $T$, Nakanishi $T$, Fujita $N$, Yamashita H (1994) Assessing myometrial invasion by endometrial carcinoma with dynamic MRI. J Comput Assist Tomogr 18:77-86

12. Nakao Y, Yokoyama M, Hara K et al (2006) MR imaging in endometrial carcinoma as a diagnostic tool for the absence of myometrial invasion. Gynecol Oncol 102:343-347

13. Seki H, Kimura M, Sakai K (1997) Myometrial invasion of endometrial carcinoma: assessment with dynamic MR and contrast-enhanced T1-weighted images. Clin Radiol 52:18-23

14. Sironi S, Colombo E, Villa G et al (1992) Myometrial invasion by endometrial carcinoma: assessment with plain and gadoliniumenhanced MR imaging. Radiology 185:207-212

15. Yamashita Y, Harada M, Sawada T, Takahashi M, Miyazaki K, Okamura H (1993) Normal uterus and FIGO stage I endometrial carcinoma: dynamic gadolinium-enhanced MR imaging. Radiology 186:495-501

16. Rockall AG, Meroni R, Sohaib SA et al (2007) Evaluation of endometrial carcinoma on magnetic resonance imaging. Int $\mathrm{J}$ Gynecol Cancer 17:188-196

17. Chung HH, Kang SB, Cho JY et al (2007) Accuracy of MR imaging for the prediction of myometrial invasion of endometrial carcinoma. Gynecol Oncol 104:654-659

18. Rechichi G, Galimberti S, Signorelli M, Perego P, Valsecchi MG, Sironi S (2009) Myometrial invasion in endometrial cancer: diagnostic performance of diffusion-weighted MR imaging at 1.5-T. Eur Radiol 20:754-762
19. Pecorelli S (2009) Revised FIGO staging for carcinoma of the vulva, cervix, and endometrium. Int J Gynaecol Obstet 105:103-104

20. Shen SH, Chiou YY, Wang JH et al (2008) Diffusion-weighted singleshot echo-planar imaging with parallel technique in assessment of endometrial cancer. AJR Am J Roentgenol 190:481-488

21. Inada $Y$, Matsuki M, Nakai G et al (2009) Body diffusionweighted MR imaging of uterine endometrial cancer: is it helpful in the detection of cancer in nonenhanced MR imaging? Eur J Radiol 70:122-127

22. Lin G, Ho KC, Wang JJ et al (2008) Detection of lymph node metastasis in cervical and uterine cancers by diffusion-weighted magnetic resonance imaging at $3 \mathrm{~T}$. J Magn Reson Imaging $28: 128-135$

23. Nakai G, Matsuki M, Inada Y et al (2008) (2008) Detection and evaluation of pelvic lymph nodes in patients with gynecologic malignancies using body diffusion-weighted magnetic resonance imaging. J Comput Assist Tomogr 32:764-768

24. Roy C, Bierry G, Matau A, Bazille G, Pasquali R (2010) Value of diffusion-weighted imaging to detect small malignant pelvic lymph nodes at $3 \mathrm{~T}$. Eur Radiol 20:1803-1811

25. Fujii S, Matsusue E, Kigawa J et al (2008) Diagnostic accuracy of the apparent diffusion coefficient in differentiating benign from malignant uterine endometrial cavity lesions: initial results. Eur Radiol 18:384-389

26. Tamai K, Koyama T, Saga T et al (2007) Diffusion-weighted MR imaging of uterine endometrial cancer. J Magn Reson Imaging $26: 682-687$ 